\title{
Laguerre Collocation Method for Solving Fredholm Integro-Differential Equations with Functional Arguments
}

\author{
Burcu Gürbüz, ${ }^{1}$ Mehmet Sezer, ${ }^{1}$ and Coşkun Güler ${ }^{2}$ \\ ${ }^{1}$ Department of Mathematics, Faculty of Science, Celal Bayar University, 45140 Manisa, Turkey \\ ${ }^{2}$ Department of Mathematical Engineering, Faculty of Chemistry-Metallurgical, Yıldiz Technical University, \\ 34220 Istanbul, Turkey
}

Correspondence should be addressed to Coşkun Güler; cguler@yildiz.edu.tr

Received 4 April 2014; Accepted 18 May 2014; Published 18 June 2014

Academic Editor: Turgut Öziş

Copyright (C) 2014 Burcu Gürbüz et al. This is an open access article distributed under the Creative Commons Attribution License, which permits unrestricted use, distribution, and reproduction in any medium, provided the original work is properly cited.

\begin{abstract}
Laguerre collocation method is applied for solving a class of the Fredholm integro-differential equations with functional arguments. This method transforms the considered problem to a matrix equation which corresponds to a system of linear algebraic equations. The reliability and efficiency of the proposed scheme are demonstrated by some numerical experiments. Also, the approximate solutions are corrected by using the residual correction method.
\end{abstract}

\section{Introduction}

Orthogonal polynomials occur often as solutions of mathematical and physical problems. They play an important role in the study of wave mechanics, heat conduction, electromagnetic theory, quantum mechanics, medicine and mathematical statistics, and so forth [1-11]. They provide a natural way to solve, expand, and interpret solutions to many types of important equations. Representation of a smooth function in terms of a series expansion using orthogonal polynomials is a fundamental concept in approximation theory and forms the basis of spectral methods of solution of Fredholm integro-differential equations with functional arguments. Laguerre polynomials $L_{n}(x)$ constitute complete orthogonal sets of functions on the semi-infinite interval $[0, \infty)$. The past couple of decades have seen a dramatic increase in the application of these models to problems in biology, physics, and engineering. In the field of Fredholm integro-differential equation with functional arguments the computation of its solution has been a great challenge and has been of great importance due to the versatility of such equations in the mathematical modeling of processes in various application fields. Mainly, we deal with the following equation:

$$
\begin{aligned}
\sum_{h=0}^{J} & \sum_{k=0}^{m} P_{h k}(x) y^{(k)}\left(\alpha_{h k} x+\beta_{h k}\right) \\
& =g(x)+\lambda \int_{a}^{b} K_{f}(x, t) y(t) d t \quad 0 \leq a \leq x, t \leq b<\infty,
\end{aligned}
$$

under the mixed conditions

$$
\sum_{k=0}^{m-1} a_{j k} y^{(k)}(0)+b_{j k} y^{(k)}(b)=\lambda_{j}, \quad j=0,1,2, \ldots, m-1,
$$

where $y(x)$ is an unknown function, the known functions $P_{h k}(x), g(x)$, and $K_{f}(x, t)$ are defined on an interval $[0, b]$, and also $a_{j k}, b_{j k}$, and $\lambda_{j}$ are appropriate constants. Our aim is to find an approximate solution expressed in the form

$$
y_{N}(x)=\sum_{n=0}^{N} a_{n} L_{n}(x)
$$


where $a_{n}$ are unknown coefficients, $L_{n}(x), n=0,1, \ldots$, are Laguerre polynomials, and $N$ is chosen positive integer. The relation between the powers of $x$ and the Laguerre polynomials $L_{n}(x)$ is

$$
L_{n}(x)=\sum_{r=0}^{n} \frac{(-1)^{r}}{r !}\left(\begin{array}{l}
n \\
r
\end{array}\right) x^{r} .
$$

To obtain a solution in the form (3) of the problem (1) under conditions (2), we can use the collocation points defined by [12-15]

$$
x_{i}=\frac{b}{N} i, \quad i=0,1,2, \ldots, N
$$

The remainder of the paper is organized as follows. Higher order linear mixed functional integro-differential equation with variable coefficients and fundamental relations is presented in Section 2. The method of finding approximate solution and the algorithm for the calculation are described in Section 3. Residual error analysis is described in Section 4. To support our finding, we present result of numerical experiments using Maple 12 in Section 5. Section 6 concludes this paper with a brief summary.

\section{Fundamental Matrix Relations}

Let us write (1) in the form

$$
D(x)=g(x)+I(x),
$$

where the functional differential part is

$$
D(x)=\sum_{h=0}^{J} \sum_{k=0}^{m} P_{h k}(x) y^{(k)}\left(\alpha_{h k} x+\beta_{h k}\right) .
$$

Fredholm integral part is

$$
I(x)=\int_{a}^{b} K_{f}(x, t) y(t) d t .
$$

We convert these parts and the mixed conditions equation (2) into the matrix form. Let us consider (1) and find the matrix forms of each term of the equation. We first consider the solution $y(x)$ and its derivative $y^{(k)}(x)$ defined by a truncated Laguerre series. Then, we can put series in the matrix form

$$
y(x)=\mathbf{L}(x) \mathbf{A}, \quad y^{(k)}(x)=\mathbf{L}^{(k)}(x) \mathbf{A},
$$

where

$$
\begin{aligned}
\mathbf{L}(x)= & {\left[\begin{array}{llll}
L_{0}(x) & L_{1}(x) & \cdots & L_{N}(x)
\end{array}\right], } \\
\mathbf{L}^{(k)}(x)= & {\left[\begin{array}{llll}
L_{0}^{(k)}(x) & L_{1}^{(k)}(x) & \cdots & L_{N}^{(k)}(x)
\end{array}\right], } \\
& \mathbf{A}=\left[\begin{array}{llll}
a_{0} & a_{1} & \cdots & a_{N}
\end{array}\right]^{T} .
\end{aligned}
$$

By using (4) and taking $n=0,1, \ldots, N$, we obtain the matrix relation as

$$
\mathbf{L}^{T}(x)=\mathbf{H} \mathbf{X}^{T}(x), \quad \mathbf{L}(x)=\mathbf{X}(x) \mathbf{H}^{T},
$$

where

$$
\mathbf{H}=\left[\begin{array}{ccccc}
\frac{(-1)^{0}}{0 !}\left(\begin{array}{l}
0 \\
0
\end{array}\right) & 0 & 0 & \ldots & 0 \\
\frac{(-1)^{0}}{0 !}\left(\begin{array}{l}
1 \\
0
\end{array}\right) & \frac{(-1)^{1}}{1 !}\left(\begin{array}{l}
1 \\
1
\end{array}\right) & 0 & \ldots & 0 \\
\frac{(-1)^{0}}{0 !}\left(\begin{array}{l}
2 \\
0
\end{array}\right) & \frac{(-1)^{1}}{1 !}\left(\begin{array}{l}
2 \\
1
\end{array}\right) & \frac{(-1)^{2}}{2 !}\left(\begin{array}{l}
2 \\
2
\end{array}\right) & \ldots & 0 \\
\vdots & \vdots & \vdots & \ddots & \vdots \\
\frac{(-1)^{0}}{0 !}\left(\begin{array}{l}
N \\
0
\end{array}\right) & \frac{(-1)^{1}}{1 !}\left(\begin{array}{l}
N \\
1
\end{array}\right) & \frac{(-1)^{2}}{2 !}\left(\begin{array}{l}
N \\
2
\end{array}\right) & \ldots & \frac{(-1)^{N}}{N !}\left(\begin{array}{l}
N \\
N
\end{array}\right)
\end{array}\right] .
$$

Then, by taking into account (11), we have

$$
\mathbf{L}^{(k)}(x)=\mathbf{X}^{(k)}(x) \mathbf{H}^{T}, \quad k=0,1,2, \ldots
$$

Moreover, it is clear that the relation between the matrix $\mathbf{X}(x)$ and its derivative $\mathbf{X}^{(k)}(x)$ is

$$
\mathbf{X}^{(k)}(x)=\mathbf{X}(x) \mathbf{B}^{k},
$$

where

$$
\mathbf{B}=\left[\begin{array}{ccccc}
0 & 1 & 0 & \cdots & 0 \\
0 & 0 & 2 & \cdots & 0 \\
\vdots & \vdots & \vdots & \ddots & \vdots \\
0 & 0 & 0 & \cdots & N \\
0 & 0 & 0 & \cdots & 0
\end{array}\right] .
$$

Hence, by means of (13) and (14) we have the matrix relation in the form

$$
\left[y^{(k)}(x)\right]=\mathbf{L}^{(k)}(x) \mathbf{A}=\mathbf{X}(x) \mathbf{B}^{k} \mathbf{H}^{T} \mathbf{A} .
$$

2.1. Matrix Representation for Differential-Difference Parts. The derivative of the matrix $\mathbf{L}(x)$ that is defined in (13), by using relation (16), can be expressed as

$$
\mathbf{L}^{(k)}(x)=\mathbf{X}(x) \mathbf{B}^{k} \mathbf{H}^{T} .
$$

By substituting $x=\alpha_{h k} x+\beta_{h k}$ into (16) [16, 17], we obtain

$$
\begin{aligned}
{\left[y^{(k)}\left(\alpha_{h k} x+\beta_{h k}\right)\right] } & =\mathbf{L}^{(k)}\left(\alpha_{h k} x+\beta_{h k}\right) \mathbf{A} \\
& =\mathbf{X}\left(\alpha_{h k} x+\beta_{h k}\right) \mathbf{B}^{k} \mathbf{H}^{T} \mathbf{A},
\end{aligned}
$$

where we have from (13)

$$
\begin{gathered}
\mathbf{L}^{(k)}\left(\alpha_{h k} x+\beta_{h k}\right)=\mathbf{X}^{(k)}\left(\alpha_{h k} x+\beta_{h k}\right) \mathbf{H}^{T}, \\
\mathbf{X}\left(\alpha_{h k} x+\beta_{h k}\right)=\left[\begin{array}{ll}
1 & \left(\alpha_{h k} x+\beta_{h k}\right)^{1} \cdots\left(\alpha_{h k} x+\beta_{h k}\right)^{N}
\end{array}\right] .
\end{gathered}
$$

Then, we consider (14) and we have

$$
\mathbf{X}^{(k)}\left(\alpha_{h k} x+\beta_{h k}\right)=\mathbf{X}(x) \mathbf{B}_{\left(\alpha_{h k}, \beta_{h k}\right)} \mathbf{B}^{k},
$$


where

$$
\mathbf{B}_{\left(\alpha_{h k}, \beta_{h k}\right)}=\left[\begin{array}{cccc}
\left(\begin{array}{l}
0 \\
0
\end{array}\right)\left(\alpha_{h k}\right)^{0}\left(\beta_{h k}\right)^{0} & \left(\begin{array}{c}
1 \\
0
\end{array}\right) & \cdots & \left(\begin{array}{c}
N \\
0
\end{array}\right)\left(\alpha_{h k}\right)^{0}\left(\beta_{h k}\right)^{N} \\
0 & \left(\begin{array}{c}
1 \\
1
\end{array}\right) & \cdots & \left(\begin{array}{c}
N \\
1
\end{array}\right)\left(\alpha_{h k}\right)^{1}\left(\beta_{h k}\right)^{N-1} \\
0 & 0 & \cdots & \left(\begin{array}{c}
N \\
2
\end{array}\right)\left(\alpha_{h k}\right)^{2}\left(\beta_{h k}\right)^{N-2} \\
\vdots & \vdots & \ddots & \vdots \\
0 & 0 & \cdots & \left(\begin{array}{c}
N \\
N
\end{array}\right)\left(\alpha_{h k}\right)^{N}\left(\beta_{h k}\right)^{0}
\end{array}\right] .
$$

Therefore, by using relation (16), the matrix representation of differential-difference part can be given by

$$
D(x)=\sum_{h=0}^{J} \sum_{k=0}^{m} \mathbf{P}_{h k}(x) \mathbf{X B}_{\left(\alpha_{h k}, \beta_{h k}\right)} \mathbf{B}^{k} \mathbf{H}^{T} \mathbf{A} .
$$

2.2. Matrix Representation for Fredholm Integral Part. $K_{f}(x, t)$ can be expanded by Laguerre series with respect to $t$ as follows:

$$
K_{f}(x, t)=\sum_{i, j=0}^{N} k_{i j}^{f}(x) L_{j}(t), \quad i, j=0,1, \ldots, N .
$$

Then, the matrix representation of the kernel function $K_{f}(x, t)$ becomes

$$
\mathbf{K}_{f}(x, t)=\mathbf{K}_{f}(x) \mathbf{L}^{T}(t),
$$

where

$$
\mathbf{K}_{f}(x, t)=\left[k_{i j}(x)\right] \quad i, j=0,1, \ldots, N .
$$

Substituting relations (9) and (24) in the Fredholm part, we obtain

$$
I(x)=\int_{a}^{b} \mathbf{X}(x) \mathbf{K}_{f} \mathbf{L}^{T} \mathbf{L} \mathbf{A} d t=\mathbf{L} \mathbf{K}_{f} \mathbf{Q A},
$$

where

$$
\begin{aligned}
\mathbf{Q} & =\int_{a}^{b} \mathbf{L}^{T} \mathbf{L} d t=\int_{a}^{b} \mathbf{H} \mathbf{X}^{T}(t) \mathbf{X}(t) \mathbf{H}^{T} d t \\
& =\mathbf{H}\left[\int_{a}^{b} \mathbf{X}^{T}(t) \mathbf{X}(t) d t\right] \mathbf{H}^{T} .
\end{aligned}
$$

We define

$$
\begin{gathered}
\mathbf{M}_{f}=\left[h_{i j}^{f}\right] \\
{\left[h_{i j}^{f}\right]=\int_{a}^{b} \mathbf{X}(t)^{T} \mathbf{X}(t) d t=\frac{b^{i+j+1}-a^{i+j+1}}{i+j+1} \quad i, j=0,1, \ldots, N .}
\end{gathered}
$$

We also have the matrix relation $\mathbf{Q}_{f}=\mathbf{H} \mathbf{M}_{f} \mathbf{H}^{T}$. Hence, the matrix representation of Fredholm integral part can be given by

$$
I(x)=\mathbf{X}(x) \mathbf{H}^{T} \mathbf{K}_{f} \mathbf{Q}_{f} \mathbf{A} .
$$

2.3. Matrix Representation of the Conditions. Let us define the matrix form of the conditions given by (2) that can be written as

$$
\sum_{k=0}^{m-1} a_{j k} y^{(k)}(0)+b_{j k} y^{(k)}(b)=\lambda_{j}, \quad j=0,1,2, \ldots, m-1 .
$$

Then, we have

$$
\begin{aligned}
{\left[y^{(k)}(0)\right] } & =\mathbf{X}(0)\left(\mathbf{B}^{T}\right)^{k} \mathbf{H}^{T} \mathbf{A}, \\
{\left[y^{(k)}(b)\right] } & =\mathbf{X}(b)\left(\mathbf{B}^{T}\right)^{k} \mathbf{H}^{T} \mathbf{A} .
\end{aligned}
$$

Briefly, we demonstrate the conditions in matrix form as

$$
\begin{array}{r}
\mathbf{U}_{j}=\sum_{k=0}^{m-1} a_{j k} y^{(k)}(0)+b_{j k} y^{(k)}(b)\left(\mathbf{B}^{T}\right)^{k} \mathbf{H}^{T} \mathbf{A}=\left[\lambda_{j}\right], \\
j, k=0,1,2, \ldots, m-1,
\end{array}
$$

where

$$
\mathbf{U}_{j} \mathbf{A}=\left[\begin{array}{llll}
u_{j 0} & u_{j 1} & \cdots & u_{j N}
\end{array}\right] .
$$

\section{Method of Solution}

We are ready to construct the fundamental matrix equation corresponding to (1). For this purpose, by substituting the matrix relations (22) and (30) into (1), we obtain

$$
\begin{array}{r}
\sum_{h=0}^{J} \sum_{k=0}^{m} \mathbf{P}_{h k}(x) \mathbf{X}(x) \mathbf{B}_{\left(\alpha_{h k}, \beta_{h k}\right)} \mathbf{B}^{k} \mathbf{H}^{T} \mathbf{A} \\
=\mathbf{G}(x)+\lambda \mathbf{X}(x) \mathbf{H}^{T} \mathbf{K}_{f} \mathbf{Q}_{f} \mathbf{A} .
\end{array}
$$

Then, we put equally spaced collocation points in (5) and relation (35) obtains

$$
\begin{array}{r}
\sum_{h=0}^{J} \sum_{k=0}^{m} \mathbf{P}_{h k}\left(x_{i}\right) \mathbf{X}\left(x_{i}\right) \mathbf{B}_{\left(\alpha_{h k}, \beta_{h k}\right)} \mathbf{B}^{k} \mathbf{H}^{T} \mathbf{A} \\
=\mathbf{G}\left(x_{i}\right)+\lambda \mathbf{X}\left(x_{i}\right) \mathbf{H}^{T} \mathbf{K}_{f} \mathbf{Q}_{f} \mathbf{A} .
\end{array}
$$

Briefly, the fundamental matrix equation is gained as follows:

$$
\left(\sum_{h=0}^{J} \sum_{k=0}^{m} \mathbf{P}_{h k} \mathbf{X} \mathbf{B}_{\left(\alpha_{h k}, \beta_{h k}\right)} \mathbf{B}^{k} \mathbf{H}^{T}-\lambda \mathbf{X} \mathbf{H}^{T} \mathbf{K}_{f} \mathbf{Q}_{f}\right) \mathbf{A}=\mathbf{G},
$$

where

$$
\begin{gathered}
\mathbf{P}_{h k}=\left[\begin{array}{ccccc}
P_{h k}\left(x_{0}\right) & 0 & 0 & \ldots & 0 \\
0 & P_{h k}\left(x_{1}\right) & 0 & \ldots & 0 \\
0 & 0 & P_{h k}\left(x_{2}\right) & \ldots & 0 \\
\vdots & \vdots & \vdots & \ddots & \vdots \\
0 & 0 & 0 & \ldots & P_{h k}\left(x_{N}\right)
\end{array}\right], \\
\mathbf{X}=\left[\begin{array}{ccccc}
1 & x_{0} & x_{0}^{2} & \ldots & x_{0}^{N} \\
1 & x_{1} & x_{1}^{2} & \ldots & x_{1}^{N} \\
1 & x_{2} & x_{2}^{2} & \ldots & x_{2}^{N} \\
\vdots & \vdots & \vdots & \ddots & \vdots \\
1 & x_{N} & x_{N}^{2} & \ldots & x_{N}^{N}
\end{array}\right], \quad \mathbf{K}_{f}=\left[\begin{array}{c}
K_{f}\left(x_{0}\right) \\
K_{f}\left(x_{1}\right) \\
K_{f}\left(x_{2}\right) \\
\vdots \\
K_{f}\left(x_{N}\right)
\end{array}\right],
\end{gathered}
$$




$$
\mathbf{G}=\left[\begin{array}{c}
g\left(x_{0}\right) \\
g\left(x_{1}\right) \\
g\left(x_{2}\right) \\
\vdots \\
g\left(x_{N}\right)
\end{array}\right]
$$

The fundamental matrix equation (35) for (1) corresponds to a system of $(N+1)$ algebraic equation for the $(N+1)$ unknown coefficients $a_{0}, a_{1}, \ldots, a_{N}[18]$. Briefly we can write (37) as

$$
\mathbf{W A}=\mathbf{G} \quad \text { or } \quad[\mathbf{W} ; \mathbf{G}]
$$

so that, for $i, j=0,1,2, \ldots, N$,

$$
\begin{aligned}
\mathbf{W} & =\left[w_{i j}\right] \\
& =\sum_{h=0}^{J} \sum_{k=0}^{m} \mathbf{P}_{h k}(x) \mathbf{X B}_{\left(\alpha_{h k}, \beta_{h k}\right)} \mathbf{B}^{k} \mathbf{H}^{T}-\lambda \mathbf{X}(x) \mathbf{H}^{T} \mathbf{K}_{f} \mathbf{Q}_{f} .
\end{aligned}
$$

Then, the matrix form of conditions (2) is

$$
\mathbf{U}_{j} \mathbf{A}=\left[\lambda_{j}\right] \quad \text { or } \quad\left[\mathbf{U}_{j} ; \lambda_{j}\right], \quad j=0,1, \ldots, m-1,
$$

where

$$
\left[\widetilde{\mathbf{U}}_{j}\right]=\left[\mathbf{U}_{j} ; \lambda_{j}\right]
$$

To obtain the solution of (1) under conditions (2), by replacing the rows in matrix (39) by the last m rows of matrix (41), we have the required augmented matrix

$$
\widetilde{\mathbf{W}} \mathbf{A}=\widetilde{\mathbf{G}} \text {. }
$$

If $\operatorname{rank}(\widetilde{\mathbf{W}})=\operatorname{rank}[\widetilde{\mathbf{W}} ; \widetilde{\mathbf{G}}]=N+1$, then we can write

$$
\mathbf{A}=(\widetilde{\mathbf{W}})^{-1} \widetilde{\mathbf{G}}
$$

Thus, the coefficients $a_{n}, i=0,1, \ldots, N$, are uniquely determined by (43) [19].

\section{Residual Correction and Error Estimation}

In this section, we give an error estimation for the Laguerre polynomial solution (3) with the residual error function, and it supports the idea of corrected Laguerre polynomial solution with respect to the residual error function. Let us define (1) with $L$ operator as follows:

$$
\begin{array}{r}
L[y(x)]=\sum_{h=0}^{J} \sum_{k=0}^{m} P_{h k}(x) y^{(k)}\left(\alpha_{h k} x+\beta_{h k}\right) \\
-\lambda \int_{a}^{b} K_{f}(x, t) y(t) d t=g(x) \\
\quad 0 \leq a \leq x, \quad t \leq b<\infty .
\end{array}
$$

Then, we get the residual function of the Laguerre collocation method as

$$
R_{N}(x)=L\left[y_{N}(x)\right]-g(x)
$$

where $y_{N}(x)$ is the approximate solution which is solved via Laguerre polynomials given by (3) of problem (1) with conditions (2). Thus, $y_{N}(x)$ satisfies the problem

$$
\begin{aligned}
& \sum_{h=0}^{J} \sum_{k=0}^{m} P_{h k}(x) y_{N}^{(k)}\left(\alpha_{h k} x+\beta_{h k}\right) \\
& =g(x)+\lambda \int_{a}^{b} K_{f}(x, t) y_{N}(t) d t \\
& 0 \leq a \leq x, \quad t \leq b<\infty,
\end{aligned}
$$

$$
\sum_{k=0}^{m-1} a_{j k} y_{N}^{(k)}(0)+b_{j k} y_{N}^{(k)}(b)=\lambda_{j}, \quad j=0,1,2, \ldots, m-1
$$

Also, the error function $E_{N}(x)$ can be defined as

$$
E_{N}(x)=y(x)-y_{N}(x),
$$

where $y(x)$ is the exact solution of problem (1) with (2) conditions. Then, by using (1), (2), (45), and (47), we obtain the error differential equation

$$
L\left[E_{N}(x)\right]=L[y(x)]-L\left[y_{N}(x)\right]=-R_{N}(x)
$$

with the homogeneous conditions

$$
\sum_{k=0}^{m-1} a_{j k} E_{N}^{(k)}(0)+b_{j k} E_{N}^{(k)}(b)=0, \quad j=0,1,2, \ldots, m-1 .
$$

Briefly, the error problem is

$$
\begin{gathered}
\sum_{h=0}^{J} \sum_{k=0}^{m} P_{h k}(x) E_{N}^{(k)}\left(\alpha_{h k} x+\beta_{h k}\right) \\
=g(x)+\lambda \int_{a}^{b} K_{f}(x, t) E_{N}(t) d t \\
0 \leq a \leq x, \quad t \leq b<\infty, \\
\sum_{k=0}^{m-1} a_{j k} E_{N}^{(k)}(0)+b_{j k} E_{N}^{(k)}(b)=0, \quad j=0,1,2, \ldots, m-1 .
\end{gathered}
$$

By solving problem (52) with the technique introduced in Section 3, we get the approximation

$$
E_{N, M}(x)=\sum_{n=0}^{M} a_{n}^{*} L_{n}(x)
$$

to $E_{N}(x)$. Consequently, by means of the polynomials $y_{N}(x)$ and $E_{N, M}(x),(M>N)$, we obtain the corrected Laguerre polynomial solution $y_{N, M}(x)=y_{N}(x)+E_{N, M}(x)$. Also, we construct the corrected error function

$$
E_{N, M}^{*}(x)=E_{N}(x)-E_{N, M}(x)=y(x)-y_{N, M}(x)
$$

and the estimated error function $E_{N, M}(x)$ [20-22]. 
4.1. Algorithm. In this section, we will consider each step of the method which is solved by Maple 12 and we distinguish the algorithms in three steps. Algorithm 1 demonstrates the calculation of approximate solution. Algorithm 2 shows each step of residual error function and its support to corrected error and corrected Laguerre polynomial solution. We plot the graphics of all functions in Algorithm 3.

Algorithm 1. Consider the following.

(1) Input initial data: the coefficients are $P_{h k}$, kernel function is $K_{f}(x, t)$, and the coefficients of the conditions are $a_{j k}, b_{j k}$. The problem is defined on $[0,1]$.

(2) Develop the algorithm by matrix inputs which are constructed by collocation points in (4).

(3) Use the conditions to construct augmented matrix and the system is done.

(4) Output: the system is solved and $A$ unknown matrix is found. Then, approximate solution is found with respect to coefficients of unknown matrix in truncated Laguerre series.

(5) End of Algorithm 1.

Algorithm 2. Consider the following.

(1) Input data: the approximate solution is $y_{N}(x)$, the error function $E_{N}(x)$, and truncated Laguerre series (3).

(2) We check for $y_{N}(x)$ that equals truncated Laguerre series. Develop the algorithm to demonstrate $R_{N}(x)$, the residual function of the Laguerre collocation method, via absolute error function $\left|E_{N}(x)\right|$.

(3) The conditions are constructed by absolute error function. Then, obtain the estimated error function $E_{N, M}$.

(4) Output: the corrected error function $E_{N, M}^{*}$ is found.

(5) End of Algorithm 2.

Algorithm 3. Consider the following.

(1) Input data: the comparison between approximate and exact solutions is demonstrated by the graphics.

(2) Output:

with(plots):

plot $1:=\operatorname{plot}(([y(x)], x=0.1)$, style $=$ point, color $=$ magenta, legend $=[$ typeset $($ "Exact Solution") $])$;

plot2 := plot $(([y(N, M)(x), x=0 . .1])$, style = line, color $=$ blue, legend $=[$ typeset $($ “Approximate Solution") $])$;

display (plot(plot1,plot2)).

(3) Input: the comparison between error and residual error solutions is demonstrated by the graphics.

(4) Output:

with(plots):

plot3 $:=\operatorname{plot}\left(\left(\left[E_{N}(x)\right], x=0.1\right)\right.$, style $=$ point, color $=$ magenta, legend $=[$ typeset $($ "Error Function") $])$; plot $:=\operatorname{plot}(([E(N, M)(x), x=0 . .1])$, style = line, color $=$ blue, legend $=[$ typeset ("Residual Error Function") $])$; display (plot(plot3,plot4)).

(5) End of Algorithm 3.

\section{Illustrative Examples}

In this section, several numerical examples are given to illustrate the accuracy and the effectiveness of the method. All of them are performed on the computer by using Maple 12.

Example 4 (see [23]). Let us first consider the functional Fredholm integro-differential equations as follows:

$$
\begin{aligned}
& y(x+1)+x y(x)-x y(x-1) \\
& =\left(2+3 x^{2}+\left(1-\frac{8}{3} e^{x}\right) x\right)+\int_{0}^{1}\left(2 x e^{x}\right) y(t) d t, \\
& 0 \leq x \leq 1,
\end{aligned}
$$

under the mixed conditions

$$
y(0)=1, \quad y\left(\frac{1}{2}\right)=\frac{5}{4},
$$

and we seek the approximate solution $y_{N}(x)$ as a truncated Laguerre series:

$$
y_{N}(x)=\sum_{r=0}^{N} a_{r} L_{r}(x), \quad 0 \leq x<\infty .
$$

Here,

$$
\begin{gathered}
\mathbf{P}_{00}(x)=x, \quad \mathbf{P}_{10}(x)=-x, \quad \mathbf{P}_{20}(x)=1, \\
g(x)=e^{x}-e+e^{-1}, \\
K_{f}(x, t)=2 x e^{x}, \\
\lambda=1 .
\end{gathered}
$$

Then, for $N=3$, the collocation points are $x_{0}=0, x_{1}=1 / 2$, $x_{2}=2 / 3$, and $x_{3}=1$ and the problem is defined by

$$
\mathbf{W}=\mathbf{P}_{00} \mathbf{X} \mathbf{H}^{T}+\mathbf{P}_{10} \mathbf{X B}_{\alpha_{1}, \beta_{1}}+\mathbf{P}_{20} \mathbf{X B}_{\alpha_{2}, \beta_{2}}-\lambda \mathbf{X H}^{T} \mathbf{K}_{f} \mathbf{Q}_{f},
$$

and matrices for conditions are

$$
\left[\begin{array}{ccc}
U_{0} & ; & \lambda_{0} \\
U_{1} & ; & \lambda_{1}
\end{array}\right]=\left[\begin{array}{cccccc}
1 & 1 & 1 & 1 & ; & 1 \\
1 & \frac{1}{2} & \frac{1}{8} & -\frac{7}{48} & ; & \frac{5}{4}
\end{array}\right],
$$

and the matrices are computed as

$$
[\mathbf{W} ; \mathbf{G}]=\left[\begin{array}{cccccc}
1 & 0 & -\frac{1}{2} & -\frac{2}{3} & ; & 2 \\
\frac{2}{27} & -\frac{61}{24} & -\frac{134}{81} & -\frac{15}{8} & ; & \frac{800}{561} \\
-\frac{41}{27} & -\frac{70}{27} & -\frac{419}{162} & -\frac{119}{54} & ; & \frac{336}{625} \\
-4 & -\frac{9}{2} & -\frac{10}{3} & -\frac{43}{24} & ; & -\frac{213}{170}
\end{array}\right] .
$$


Then, we have the augmented matrix

$$
[\widetilde{\mathbf{W}} ; \widetilde{\mathbf{G}}]=\left[\begin{array}{cccccc}
1 & 0 & -\frac{1}{2} & -\frac{2}{3} & ; & 2 \\
\frac{2}{27} & -\frac{61}{24} & -\frac{134}{81} & -\frac{15}{8} & ; & \frac{800}{561} \\
1 & 1 & 1 & 1 & ; & 1 \\
1 & \frac{1}{2} & \frac{7}{8} & -\frac{7}{48} & ; & \frac{5}{4}
\end{array}\right]
$$

Subsequently, we have

$$
\mathbf{A}=\left[\begin{array}{llll}
\frac{423}{142} & -\frac{545}{140} & \frac{360}{190} & \frac{583}{122}
\end{array}\right]
$$

and we obtain the approximate solution of the problem for $N=3$ as

$$
\begin{aligned}
y_{3}(x) & \\
& =0.999999999999996-0.366738338031 .10^{-3} x \\
& +1.01100215014091 x^{2}-0.733476676060432 .10^{-3} x^{3} .
\end{aligned}
$$

We follow the same steps for $N=10$ and $N=15$ :

$$
\begin{aligned}
y_{10}(x)= & -0.12404861 .10^{-6} x+1.000000772576 x^{2} \\
& +0.999999999999973-0.896642 .10^{-6} x^{3} \\
& -0.12907822 .10^{-5} x^{4}+0.195475243 .10^{-5} x^{5} \\
& +0.709505509 .10^{-6} x^{6}-0.14545410555 .10^{-5} x^{7} \\
& +0.39178123286 .10^{-7} x^{8} \\
& +0.3995801024017 .10^{-6} x^{9} \\
& -0.109576123674046 .10^{-6} x^{10}
\end{aligned}
$$

and for $N=15$

$$
\begin{aligned}
y_{15}(x)= & 0.99999999945109 x^{2}+0.33996 .10^{-9} x \\
& +1.00000000000001-0.135090 .10^{-8} x^{3} \\
& +0.2063949 .10^{-8} x^{4}+0.14857110 .10^{-8} x^{5} \\
& -0.26538783 .10^{-8} x^{6}-0.386318369 .10^{-9} x^{7} \\
& +0.153862741670 .10^{-8} x^{8} \\
& -0.311133726104 .10^{-9} x^{9} \\
& -0.32406068899445 .10^{-9} x^{10}
\end{aligned}
$$

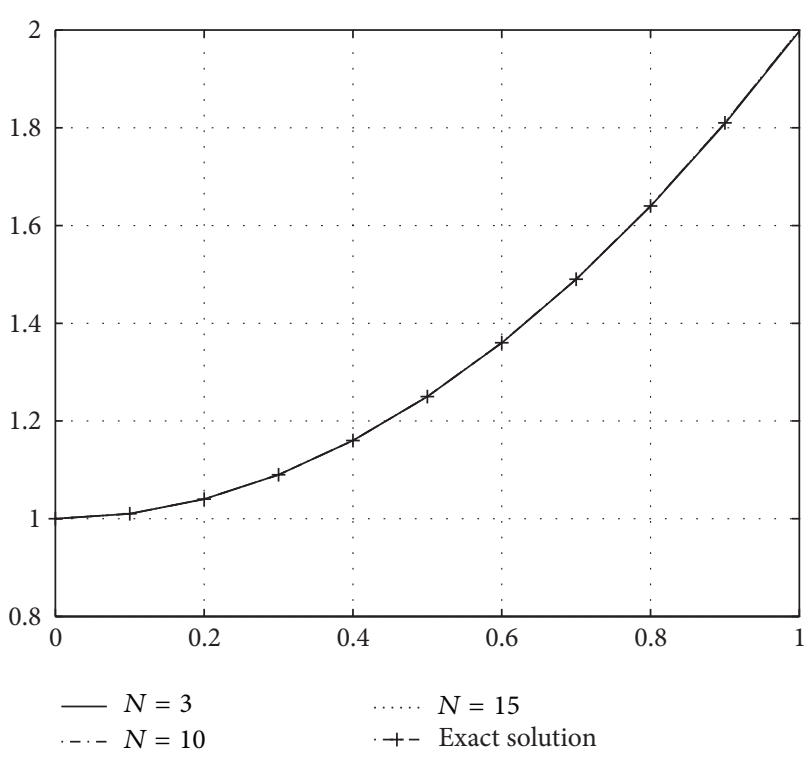

Figure 1: Numerical and exact solution of Example 4 for $N=$ $3,10,15$.

$$
\begin{aligned}
& +0.1760808732067 .10^{-9} x^{11} \\
& -0.30984313933219 .10^{-10} x^{12} \\
& +0.187639980825480 .10^{-11} x^{13} \\
& -0.458623284005214 .10^{-13} x^{14} \\
& +0.399275908003723 .10^{-15} x^{15}
\end{aligned}
$$

The exact solution of this problem is $y(x)=x^{2}+1$.

Table 1 shows the comparison between exact and approximate solutions for $N=3,10,15$ values. The results of residual error function support the evaluation of the corrected new Laguerre polynomial solutions for different $N$ and $M$ values. Figure 1 shows us the comparison between exact and approximate solutions for various $N$ values. Figure 2 shows the absolute error and absolute residual error functions. Mainly, this comparison supports the idea of corrected Laguerre polynomial solution for different $N$ and $M$ values.

Table 2 shows the corrected absolute errors by our method for $N=3,10,15$ and $M=7,12,20$. The results support the idea that when $N$ and $M$ values are chosen large enough, the absolute error and residual error are decreased.

Example 5 (see [24]). Let us find the Laguerre series solution of the following second order linear functional Fredholm integro-differential equation with variable coefficients:

$$
\begin{array}{r}
x^{2} y^{\prime \prime}(x)+x y^{\prime}(x)+y(x)+y(x-1) \\
=\int_{0}^{1}\left(\frac{12}{7} x^{2}-2\right) y(t) d t+2 x^{2}-\frac{28}{3} x+13, \\
0 \leq x \leq 1,
\end{array}
$$


TABLE 1: Numerical solution of Example 4 for different $N$ values.

\begin{tabular}{lccccccc}
\hline & & \multicolumn{2}{c}{ Present method and corrected Laguerre polynomial solution } & \\
$x$ & Exact solution & $y_{3}$ & $y_{3,7}$ & $y_{10}$ & $y_{10,12}$ & $y_{15}$ \\
\hline 0.0 & 1.000000 & 0.999999 & 0.99999 & 0.999999 & 1.00000 & 1.000000 & 1.000000 \\
0.1 & 1.010000 & 1.009736 & 1.01054 & 1.009999 & 1.00999 & 1.010000 & 1.010000 \\
0.2 & 1.040000 & 1.039648 & 1.04075 & 1.039999 & 1.03999 & 1.040000 & 1.040000 \\
0.3 & 1.090000 & 1.089692 & 1.09068 & 1.090000 & 1.08999 & 1.090000 & 1.090000 \\
0.4 & 1.160000 & 1.159824 & 1.16040 & 1.160000 & 1.15999 & 1.160000 \\
0.5 & 1.250000 & 1.249999 & 1.25000 & 1.250000 & 1.24999 & 1.250000 & 1.160000 \\
0.6 & 1.360000 & 1.360176 & 1.35957 & 1.359999 & 1.36000 & 1.359999 \\
0.7 & 1.490000 & 1.490308 & 1.48923 & 1.489999 & 1.49000 & 1.489999 \\
0.8 & 1.640000 & 1.640352 & 1.63909 & 1.639999 & 1.64000 & 1.639999 \\
0.9 & 1.810000 & 1.810264 & 1.80931 & 1.809999 & 1.80999 & 1.809999 \\
1.0 & 2.000000 & 2.000000 & 2.00202 & 2.000000 & 1.99999 & 1.9999999 \\
\hline
\end{tabular}

TABle 2: Comparison of the actual and estimated absolute errors for $N=3,10,15$ and $M=7,12,20$ of Example 4.

\begin{tabular}{|c|c|c|c|c|c|c|}
\hline \multicolumn{7}{|c|}{ The actual absolute and estimated absolute errors } \\
\hline$x$ & $E_{3}$ & $E_{3,7}$ & $E_{10}$ & $E_{10,12}$ & $E_{15}$ & $E_{15,20}$ \\
\hline 0.0 & $0.40000 E-14$ & $0.48000 E-08$ & $0.27000 E-13$ & $0.13300 E-09$ & $0.1000 E-13$ & $0.34760 E-13$ \\
\hline 0.1 & $0.26405 E-03$ & $0.54125 E-03$ & $0.56847 E-08$ & $0.11292 E-07$ & $0.2738 E-10$ & $0.10459 E-09$ \\
\hline 0.2 & $0.35206 E-03$ & $0.74827 E-04$ & $0.24926 E-08$ & $0.16453 E-07$ & $0.3885 E-10$ & $0.15989 E-09$ \\
\hline 0.3 & $0.30806 E-03$ & $0.67809 E-03$ & $0.26114 E-08$ & $0.14766 E-07$ & $0.3452 E-10$ & $0.16136 E-09$ \\
\hline 0.4 & $0.17603 E-03$ & $0.40076 E-03$ & $0.42220 E-08$ & $0.82377 E-08$ & $0.1916 E-10$ & $0.10735 E-09$ \\
\hline 0.5 & $0.10000 E-13$ & $0.28387 E-09$ & $0.21000 E-12$ & $0.50360 E-09$ & 0.00000000 & $0.26070 E-13$ \\
\hline 0.6 & $0.17603 E-03$ & $0.42664 E-03$ & $0.88534 E-08$ & $0.47667 E-08$ & $0.1571 E-10$ & $0.15464 E-09$ \\
\hline 0.7 & $0.30806 E-03$ & $0.76824 E-03$ & $0.18229 E-07$ & $0.54107 E-08$ & $0.2306 E-10$ & $0.34641 E-09$ \\
\hline 0.8 & $0.35207 E-03$ & $0.90156 E-03$ & $0.22651 E-07$ & $0.18877 E-08$ & $0.2078 E-10$ & $0.56197 E-09$ \\
\hline 0.9 & $0.26405 E-03$ & $0.69278 E-03$ & $0.17309 E-07$ & $0.23159 E-08$ & $0.1137 E-10$ & $0.78552 E-09$ \\
\hline 1.0 & 0.000000000 & $0.12445 E-07$ & $0.15600 E-11$ & $0.11339 E-08$ & $0.1000 E-13$ & $0.10001 E-08$ \\
\hline
\end{tabular}

TABLE 3: Numerical solution of Example 5 for different $N$ values.

\begin{tabular}{|c|c|c|c|c|c|c|c|}
\hline \multicolumn{8}{|c|}{ Present method and corrected Laguerre polynomial solution } \\
\hline$x$ & Exact solution & $y_{8}$ & $y_{8,10}$ & $y_{10}$ & $y_{10,12}$ & $y_{18}$ & $y_{18,22}$ \\
\hline 0.0 & 4.000000 & 4.000000 & 4.000000 & 3.999999 & 3.999999 & 4.000000 & 4.000000 \\
\hline 0.1 & 3.610000 & 3.607781 & 3.608978 & 3.607685 & 3.610898 & 3.610363 & 3.608798 \\
\hline 0.2 & 3.240000 & 3.230410 & 3.235483 & 3.230156 & 3.243601 & 3.240185 & 3.235706 \\
\hline 0.3 & 2.890000 & 2.867005 & 2.878938 & 2.866678 & 2.898090 & 2.887697 & 2.881383 \\
\hline 0.4 & 2.560000 & 2.516891 & 2.538843 & 2.516663 & 2.574315 & 2.551308 & 2.546356 \\
\hline 0.5 & 2.250000 & 2.179554 & 2.214765 & 2.179633 & 2.272214 & 2.229593 & 2.231063 \\
\hline 0.6 & 1.960000 & 1.854596 & 1.906326 & 1.855192 & 1.991717 & 1.921284 & 1.935850 \\
\hline 0.7 & 1.690000 & 1.541700 & 1.613200 & 1.543010 & 1.732756 & 1.625252 & 1.661011 \\
\hline 0.8 & 1.440000 & 1.240609 & 1.335103 & 1.242812 & 1.495265 & 1.340495 & 1.406788 \\
\hline 0.9 & 1.210000 & 0.951112 & 1.071789 & 0.954366 & 1.279181 & 1.066119 & 1.173387 \\
\hline 1.0 & 1.000000 & 0.673025 & 0.823042 & 0.677475 & 1.084448 & 0.801327 & 0.960984 \\
\hline
\end{tabular}

with initial conditions $y(0)=4, y^{\prime}(0)=-4$ and its exact solution is $y(x)=(x-2)^{2}$. We obtained the approximate solution of the problem for $N=8,10,18$ and the matrices are computed. Hence linear algebraic system is gained. This system is approximately solved using Maple 12.
Table 3 shows us the comparison between exact solution, approximate solutions for different $N$ values, and corrected Laguerre polynomial solutions with respect to the residual error analysis. Figure 3 shows us the comparison between exact and approximate solutions for different $N$ values. 
TABLE 4: Comparison of the actual and estimated absolute errors for $N=8,10,18$ and $M=10,12,22$ of Example 5.

\begin{tabular}{lcccccc}
\hline \multicolumn{7}{c}{ The actual absolute and estimated absolute errors } \\
$x$ & $E_{8}$ & $E_{8,10}$ & $E_{10}$ & $E_{10,12}$ & $E_{18}$ \\
\hline 0.0 & $0.67000 E-15$ & $0.34000 E-13$ & $0.12300 E-12$ & $0.82600 E-11$ & $0.33307 E-08$ & $0.10950 E-09$ \\
0.1 & $0.22193 E-02$ & $0.10221 E-02$ & $0.39880 E-04$ & $0.89756 E-03$ & $0.36289 E-03$ & $0.12021 E-02$ \\
0.2 & $0.95897 E-02$ & $0.45169 E-02$ & $0.26746 E-04$ & $0.36009 E-02$ & $0.18489 E-03$ & $0.42936 E-02$ \\
0.3 & $0.22995 E-01$ & $0.11062 E-01$ & $0.22276 E-03$ & $0.80900 E-02$ & $0.23027 E-02$ & $0.86169 E-02$ \\
0.4 & $0.43109 E-01$ & $0.21157 E-01$ & $0.87128 E-03$ & $0.14315 E-01$ & $0.86922 E-02$ & $0.13641 E-01$ \\
0.5 & $0.70446 E-01$ & $0.35235 E-01$ & $0.20625 E-02$ & $0.22214 E-01$ & $0.20407 E-01$ & $0.18937 E-01$ \\
0.6 & $0.10540 E-01$ & $0.53674 E-01$ & $0.39232 E-02$ & $0.31717 E-01$ & $0.38716 E-01$ & $0.24149 E-01$ \\
0.7 & $0.14829 E-01$ & $0.76799 E-01$ & $0.65657 E-02$ & $0.42756 E-01$ & $0.64748 E-01$ & $0.28989 E-01$ \\
0.8 & $0.19939 E-01$ & $0.10489 E-01$ & $0.10089 E-01$ & $0.55265 E-01$ & $0.99505 E-01$ & $0.33212 E-01$ \\
0.9 & $0.25889 E-01$ & $0.13821 E-01$ & $0.14583 E-01$ & $0.69182 E-01$ & $0.14389 E-01$ & $0.36613 E-01$ \\
1.0 & $0.32697 E-01$ & $0.17696 E-01$ & $0.20124 E-01$ & $0.84448 E-01$ & $0.19867 E-01$ & $0.39016 E-01$ \\
\hline
\end{tabular}

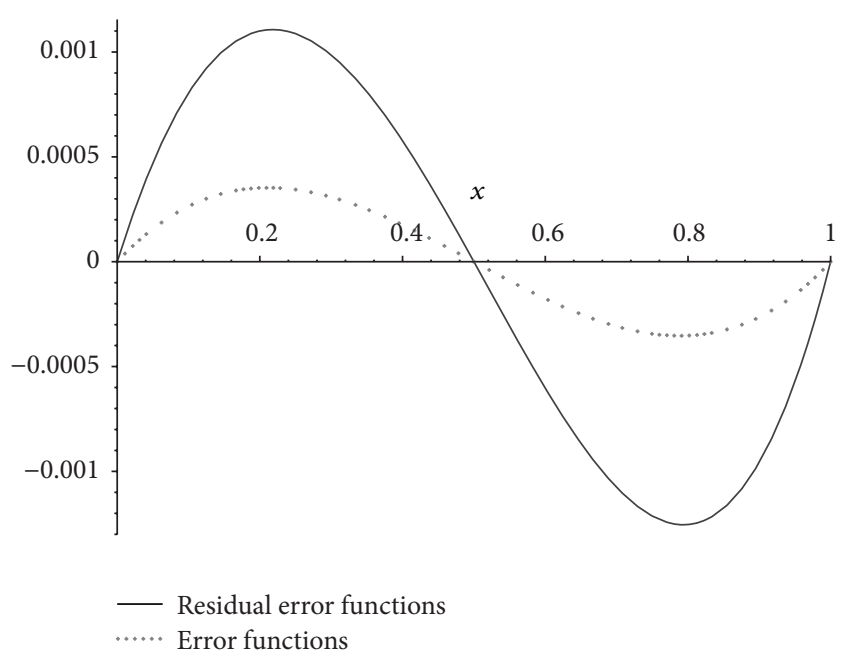

FigURE 2: Comparison of error and residual error functions for $y_{3,7}$ values for Example 4.

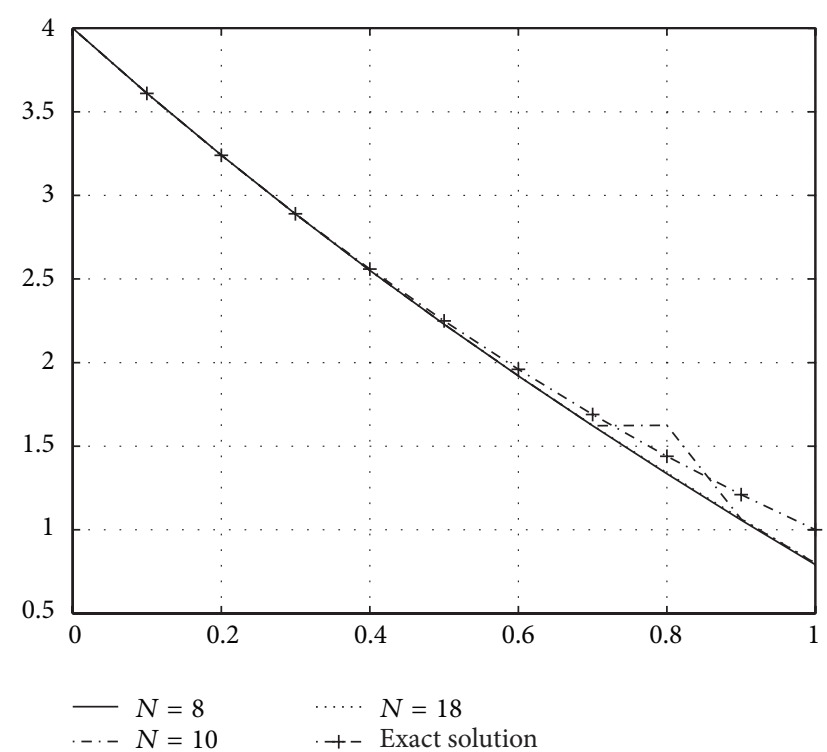

FIGURE 3: Numerical and exact solution of Example 5 for $N=8,10$, 18.

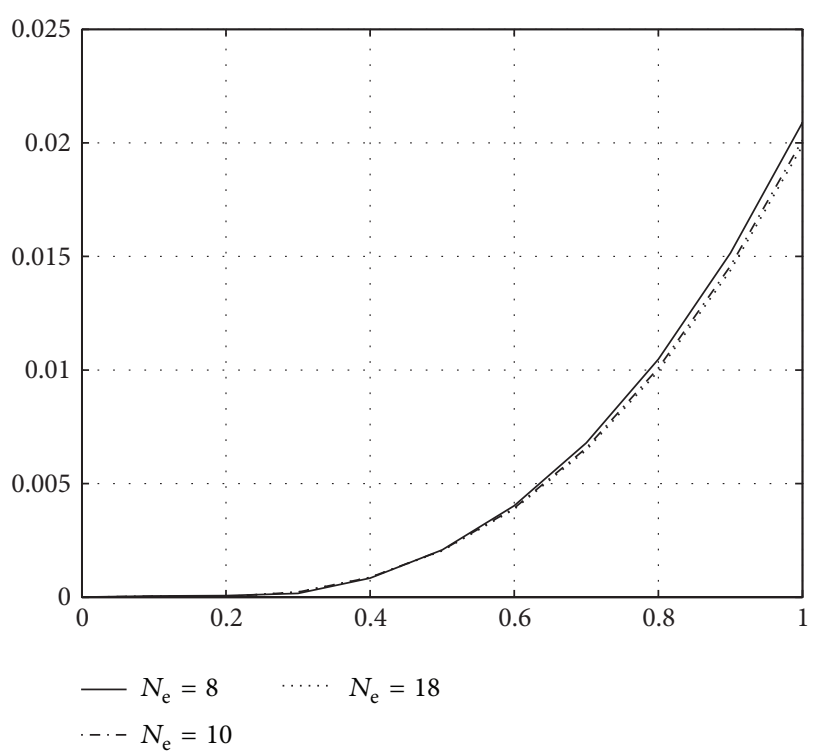

FIgURE 4: Error function of Example 5 for various $N$ values.

Figure 4 shows the comparison of absolute error function of Example 5 for the same $N$ values.

Figure 5 shows us the comparison between absolute error and absolute residual error graphics.

Table 4 shows the corrected absolute errors by our method for $N=8,10,18$ and $M=10,12,22$.

Example 6 (see [25]). Let us find the Laguerre series solution of the following second order linear functional Fredholm integro-differential equation with variable coefficients:

$$
\begin{aligned}
(x+ & 4)^{2} y^{\prime \prime}(x)-(x+4) y^{\prime}(x)+y(x-1)+y^{\prime}(x-1) \\
= & \ln (x+3)+\frac{1}{x+3}+3 \ln (3)-5 \ln (5) \\
& +\int_{-1}^{1} y(t) d t, \quad 0 \leq x \leq 1 .
\end{aligned}
$$




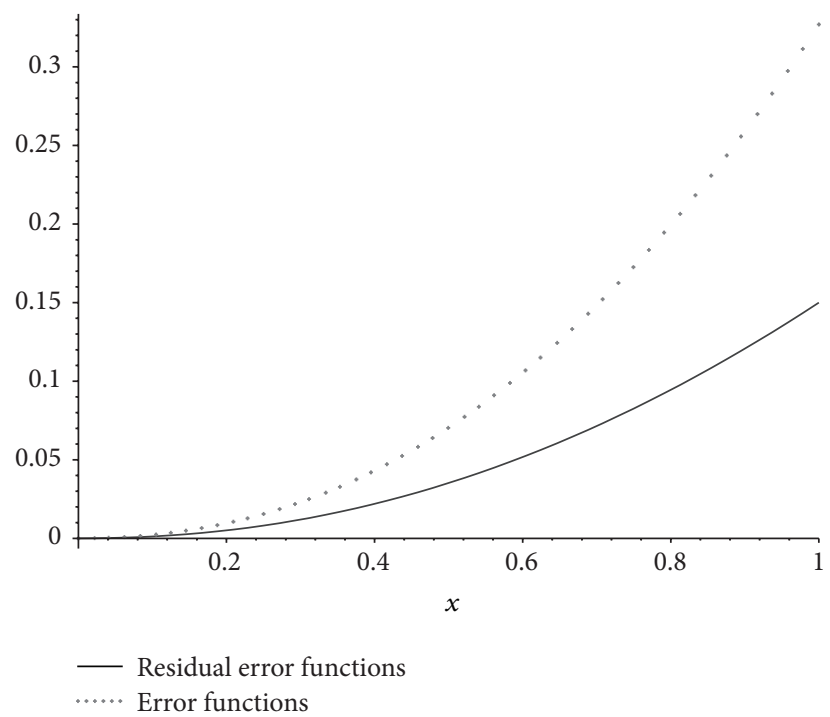

Figure 5: Comparison of error and residual error functions for $y_{8,10}$ values.

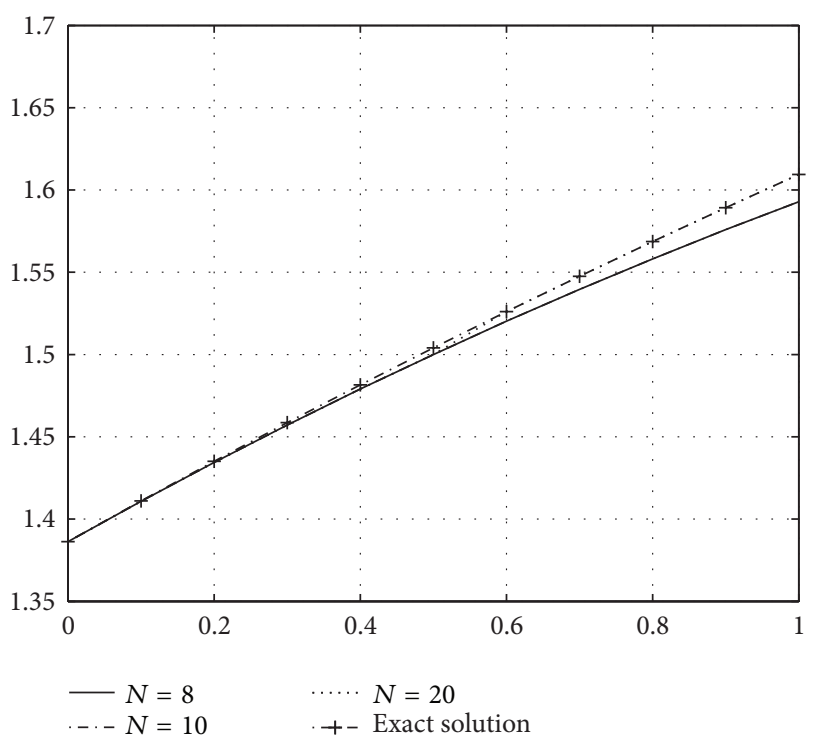

FIGURE 6: Numerical and exact solution of Example 6 for $N=8,10$, 20.

with initial conditions $y(0)=\ln (4)$ and $y^{\prime}(0)=1 / 4$ and its exact solution is $y(x)=\ln (x+4)$. We obtained the approximate solution of the problem for $N=8, N=10$, and $N=20$ which are tabulated and graphed.

Table 5 shows the exact solution comparison with approximate solutions for $N=8,10,20$ with present method. Absolute error comparisons are also given in the same table. Figure 6 shows the comparison between approximate solutions for different $N$ values. We observe the absolute error differences for the same $N$ values in Figure 7.

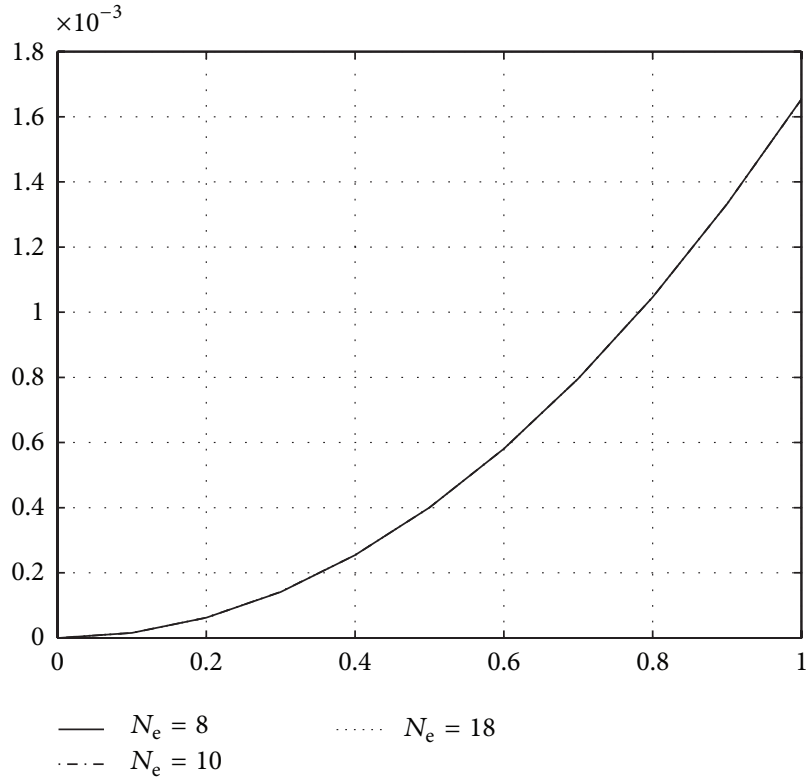

Figure 7: Error function of Example 6 for various $N$ values, $N_{\mathrm{e}}=$ $8,10,20$.

Example 7 (see [24]). Consider the linear functional Fredholm integro-differential equation

$$
\begin{aligned}
& y^{\prime \prime}(x)+x y^{\prime}(x)+x y(x)-y^{\prime}(x-1)+y(x-1) \\
& \quad=x(\sin (x)+\cos (x))-\cos (x) \\
& \quad+\sin (x-1)+\cos (x-1) \\
& \quad+4 x \sin (1) \int_{-1}^{1}(3 t-2 x) y(t) d t, \quad 0 \leq x \leq 1,
\end{aligned}
$$

with conditions $y(0)=1, y^{\prime}(0)=0$ and exact solution $y(x)=$ $\cos (x)$.

Table 6 shows the comparison between exact and approximate solutions and corrected Laguerre polynomial solutions for the different $N$ and $M$ values. Figure 8 shows the comparison of absolute error functions for the same $N$ values.

Table 7 shows the comparison between exact and approximate solutions and corrected Laguerre polynomial solutions for the different $N$ and $M$ values.

\section{Conclusion}

In recent years, the studies of linear functional Fredholm integro-differential equation have attracted the attention of many mathematicians and physicists [26-28]. Laguerre collocation methods are used to solve the linear integrodifferential equation with functional arguments numerically. It is observed that the method has the best advantage when the known functions in equation can be expanded to Laguerre series. Another advantage of the method is that the Laguerre polynomial coefficients of the solution are found very easily by using computer programs. Shorter 
TABLE 5: Numerical solution and absolute error of Example 6 for different $N$ values.

\begin{tabular}{|c|c|c|c|c|c|c|c|}
\hline \multicolumn{8}{|c|}{ Present method } \\
\hline$x$ & Exact solution & $y_{8}$ & $E_{8}$ & $y_{10}$ & $E_{10}$ & $y_{20}$ & $E_{20}$ \\
\hline 0.0 & 1.386294 & 1.386294 & 0.00000000 & 1.386294 & 0.00000000 & 1.386294 & $0.1200 E-17$ \\
\hline 0.1 & 1.410987 & 1.410833 & $0.15424 E-4$ & 1.410833 & $0.15424 E-4$ & 1.410833 & $0.15424 E-4$ \\
\hline 0.2 & 1.435085 & 1.434461 & $0.62350 E-4$ & 1.434461 & $0.62350 E-4$ & 1.434461 & $0.62350 E-4$ \\
\hline 0.3 & 1.458615 & 1.457198 & $0.14110 E-3$ & 1.457198 & $0.14165 E-3$ & 1.457198 & $0.14165 E-3$ \\
\hline 0.4 & 1.481605 & 1.479064 & $0.25409 E-3$ & 1.479064 & $0.25409 E-3$ & 1.479064 & $0.25409 E-3$ \\
\hline 0.5 & 1.504077 & 1.500007 & $0.40029 E-3$ & 1.500007 & $0.40029 E-3$ & 1.500074 & $0.40029 E-3$ \\
\hline 0.6 & 1.526056 & 1.520248 & $0.58081 E-3$ & 1.520248 & $0.58081 E-3$ & 1.526056 & $0.58081 E-3$ \\
\hline 0.7 & 1.547563 & 1.539602 & $0.79609 E-3$ & 1.539602 & $0.79609 E-3$ & 1.547563 & $0.79609 E-3$ \\
\hline 0.8 & 1.568616 & 1.558151 & $0.10465 E-2$ & 1.558151 & $0.10465 E-2$ & 1.568616 & $0.10465 E-2$ \\
\hline 0.9 & 1.589235 & 1.575911 & $0.13324 E-2$ & 1.575911 & $0.13324 E-2$ & 1.589235 & $0.13324 E-2$ \\
\hline 1.0 & 1.609438 & 1.592899 & $0.16540 E-2$ & 1.592899 & $0.16539 E-2$ & 1.609438 & $0.16539 E-2$ \\
\hline
\end{tabular}

TABLE 6: Numerical solution of Example 7 for different $N$ values.

\begin{tabular}{|c|c|c|c|c|c|c|c|}
\hline \multicolumn{8}{|c|}{ Present method and corrected Laguerre polynomial solution } \\
\hline$x$ & Exact solution & $y_{12}$ & $y_{12,15}$ & $y_{22}$ & $y_{22,28}$ & $y_{25}$ & $y_{25,28}$ \\
\hline 0.0 & 1.000000 & 0.999999 & 1.000000 & 0.999999 & 1.000000 & 1.000000 & 1.000000 \\
\hline 0.1 & 0.995004 & 0.996593 & 0.996600 & 0.996593 & 0.993964 & 0.996593 & 0.993963 \\
\hline 0.2 & 0.980067 & 0.986641 & 0.986365 & 0.986641 & 0.975909 & 0.986641 & 0.975909 \\
\hline 0.3 & 0.955336 & 0.969544 & 0.969444 & 0.969544 & 0.946004 & 0.969544 & 0.946003 \\
\hline 0.4 & 0.921061 & 0.946139 & 0.946036 & 0.946139 & 0.904531 & 0.946139 & 0.904529 \\
\hline 0.5 & 0.877583 & 0.916382 & 0.916381 & 0.916382 & 0.851886 & 0.916382 & 0.851884 \\
\hline 0.6 & 0.825336 & 0.880504 & 0.880768 & 0.880504 & 0.788573 & 0.880504 & 0.788570 \\
\hline 0.7 & 0.764842 & 0.838776 & 0.839522 & 0.838776 & 0.715199 & 0.838776 & 0.715195 \\
\hline 0.8 & 0.696707 & 0.791509 & 0.793010 & 0.791509 & 0.632466 & 0.791509 & 0.632461 \\
\hline 0.9 & 0.621610 & 0.739046 & 0.741629 & 0.739046 & 0.541167 & 0.739046 & 0.541161 \\
\hline 1.0 & 0.540302 & 0.681764 & 0.685810 & 0.681764 & 0.442174 & 0.681764 & 0.442167 \\
\hline
\end{tabular}

TABLE 7: Comparison of the actual and estimated absolute errors for $N=12,22,25$ and $M=15,28,28$ of Example 7.

\begin{tabular}{lcccccc}
\hline \multicolumn{7}{c}{ The actual absolute and estimated absolute errors } \\
$x$ & $E_{12}$ & $E_{12,15}$ & $E_{22}$ & $E_{22,28}$ & $E_{25}$ \\
\hline 0.0 & $0.69500 E-17$ & $7.000000 E-13$ & $0.10000 E-18$ & 0.0000000000 & 0.000000000 & 0.0000000000 \\
0.1 & $0.15886 E-03$ & $3.681538 E-06$ & $0.15886 E-03$ & $1.040134 E-03$ & $0.15886 E-03$ & $1.040221 E-03$ \\
0.2 & $0.63428 E-03$ & $2.207214 E-05$ & $0.63428 E-03$ & $4.157153 E-03$ & $0.63428 E-03$ & $4.157504 E-03$ \\
0.3 & $0.14208 E-02$ & $4.966673 E-05$ & $0.14208 E-02$ & $9.332459 E-03$ & $0.14208 E-02$ & $9.333242 E-03$ \\
0.4 & $0.25078 E-02$ & $5.150761 E-05$ & $0.25078 E-02$ & $1.652982 E-02$ & $0.25078 E-02$ & $1.653119 E-02$ \\
0.5 & $0.38799 E-02$ & $2.543790 E-11$ & $0.38799 E-02$ & $2.569605 E-02$ & $0.38799 E-02$ & $2.569816 E-02$ \\
0.6 & $0.55168 E-02$ & $1.324508 E-04$ & $0.55168 E-02$ & $3.676180 E-02$ & $0.55168 E-02$ & $3.676477 E-02$ \\
0.7 & $0.73934 E-02$ & $3.734395 E-04$ & $0.73934 E-02$ & $4.964259 E-02$ & $0.73934 E-02$ & $4.964652 E-02$ \\
0.8 & $0.94802 E-02$ & $7.505606 E-04$ & $0.94802 E-02$ & $6.423988 E-02$ & $0.94802 E-02$ & $6.424488 E-02$ \\
0.9 & $0.11744 E-01$ & $1.291408 E-03$ & $0.11744 E-01$ & $8.044241 E-02$ & $0.11744 E-01$ & $8.044855 E-02$ \\
1.0 & $0.14146 E-01$ & $2.023575 E-03$ & $0.14146 E-01$ & $9.812752 E-02$ & $0.14146 E-01$ & $9.813488 E-02$ \\
\hline
\end{tabular}

computation time and lower operation count result in reduction of cumulative truncation errors and improvement of overall accuracy. To get the best approximating solution of the equation, we have improved the Laguerre collocation method with the aid of the residual error function for solving the linear Fredholm integro-differential equation with piecewise intervals. For this purpose, the power of the employed method is confirmed. As a result, considerable solutions are shown in tables and figures. We assured the correctness of the obtained solutions by putting them back into the original equation with the aid of Maple 12. As a future work, the method can also be extended to the system 


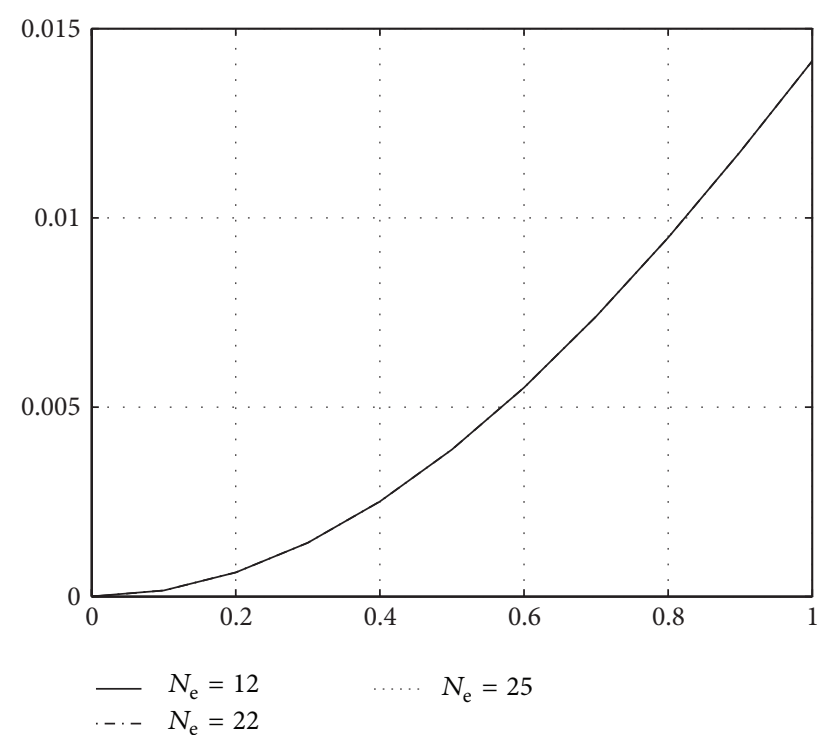

FIGURE 8: Numerical and exact solution of Example 7 for $N=12$, $22,25$.

of linear functional Fredholm integro-differential equations with variable coefficients and their residual error analysis, but some modifications are required.

\section{Conflict of Interests}

The authors declare that there is no conflict of interests regarding the publication of this paper.

\section{References}

[1] R. P. Agarwal, Ed., Contributions in Numerical Mathematics, World Scientific Publishing, Singapore, 1993.

[2] R. P. Agarwal, Ed., Dynamical Systems and Applications, World Scientific Publishing, Singapore, 1995.

[3] J. Dieudonné, Orthogonal Polynomials and Applications, 1985.

[4] S. Chandrasekhar, An Introduction to the Study of Stellar Structure, Dover Publications, New York, NY, USA, 1957.

[5] J. Rashidinia, A. Tahmasebi, and S. Rahmany, "A reliable treatment for nonlinear Volterra integro-differential," Journal of Information and Computing Science, vol. 9, no. 1, pp. 3-10, 2014.

[6] Z. Chen and X. Cheng, "An efficient algorithm for solving Fredholm integro-differential equations with weakly singular kernels," Journal of Computational and Applied Mathematics, vol. 257, pp. 57-64, 2014.

[7] J. G. Pipe and N. R. Zwart, "Spiral trajectory design: a flexible numerical algorithm and base analytical equations," Magnetic Resonance in Medicine, vol. 71, pp. 278-285, 2014.

[8] R. Farnoosh and M. Ebrahimi, "Monte Carlo method for solving Fredholm integral equations of the second kind," Applied Mathematics and Computation, vol. 195, no. 1, pp. 309-315, 2008.

[9] A. Ghosh, R. Elber, and H. A. Scheraga, "An atomically detailed study of the folding pathways of protein A with the stochastic difference equation," Proceedings of the National Academy of Sciences of the United States of America, vol. 99, no. 16, pp. 1039410398, 2002.
[10] K. Wang and Q. Wang, "Taylor collocation method and conveergence analysis for the Volterra-Fredholm integral equations," Journal of Computational and Applied Mathematics, vol. 260, pp. 294-300, 2014.

[11] A. Ovchinnikov, "Difference integrability conditions for parameterized linear difference and differential equations," Advances in Applied Mathematics, vol. 53, pp. 61-71, 2014.

[12] G. A. Andrews, R. Askey, and R. Roy, Special Functions, Cambridge University Press, Cambridge, UK, 2000.

[13] P. K. Sahu and S. Saha Ray, "Numerical solutions for the system of Fredholm integral equations of second kind by a new approach involving semiorthogonal B-spline wavelet collocation method," Applied Mathematics and Computation, vol. 234, pp. 368-379, 2014.

[14] S. Sedaghat, Y. Ordokhani, and M. Dehghan, "On spectral method for Volterra functional integro-differential equations of neutral type," Numerical Functional Analysis and Optimization, vol. 35, no. 2, pp. 223-239, 2014.

[15] S. Mashayekhi, M. Razzaghi, and O. Tripak, "Solution of the nonlinear mixed Volterra-Fredholm integral equations by hybrid of blockpulse functions and Bernoulli polynomials," The Scientific World Journal, vol. 2014, Article ID 413623, 8 pages, 2014.

[16] M. Gülsu, Y. Öztürk, and M. Sezer, "Numerical approach for solving Volterra integro differential equations with piecewise intervals," Journal of Advanced Research in Applied Mathematics, vol. 4, no. 1, pp. 23-37, 2012.

[17] M. Gülsu and Y. Öztürk, "Numerical approach for the solution of hypersingular integro-differential equations," Applied Mathematics and Computation, vol. 230, pp. 701-710, 2014.

[18] B. Gürbüz, M. Gülsu, and M. Sezer, "Numerical approach of high-order linear delay difference equations with variable coefficients in terms of Laguerre polynomials," Mathematical and Computational Applications, vol. 16, no. 1, pp. 267-278, 2011.

[19] A. Akyüz-Daşcioğlu and H. Çerdik-Yaslan, "The solution of high-order nonlinear ordinary differential equations by Chebyshev series," Applied Mathematics and Computation, vol. 217, no. 12, pp. 5658-5666, 2011.

[20] K. Erdem, S. Yalçinbaş, and M. Sezer, "A Bernoulli polynomial approach with residual correction for solving mixed linear Fredholm integro-differential-difference equations," Journal of Difference Equations and Applications, vol. 19, no. 10, pp. 16191631, 2013.

[21] Ş. Yüzbaşı, E. Gök, and M. Sezer, "Müntz-Legendre polynomial solutions of linear delay Fredholm integro-differential equations and residual correction," Mathematical and Computational Applications, vol. 18, no. 3, pp. 476-485, 2013.

[22] Ş. Yüzbaşı and M. Sezer, "An improved Bessel collocation method with a residual error function to solve a class of LaneEmden differential equations," Mathematical and Computer Modelling, vol. 57, no. 5-6, pp. 1298-1311, 2013.

[23] M. Sezer and M. Gülsu, "A new polynomial approach for solving difference and Fredholm integro-difference equations with mixed argument," Applied Mathematics and Computation, vol. 171, no. 1, pp. 332-344, 2005.

[24] M. Gülsu and Y. Öztürk, "A new collocation method for solution of mixed linear integro-differential-difference equations," Applied Mathematics and Computation, vol. 216, no. 7, pp. 21832198, 2010.

[25] S. Yalçinbaş and T. Akkaya, "A numerical approach for solving linear integro-differential-difference equations with Boubaker 
polynomial bases," Ain Shams Engineering Journal, vol. 3, no. 2, pp. 153-161, 2012.

[26] E. H. Doha, D. Baleanu, A. H. Bhrawy, and M. A. Abdelkawy, "A Jacobi collocation method for solving nonlinear Burgers-type equations," Abstract and Applied Analysis, vol. 2013, Article ID 760542, 12 pages, 2013.

[27] S. Alavi and A. Heydari, "An Analytic approximate solution of the matrix Riccati differential equation arising from the LQ optimal control problems," Journal of Advances in Mathematics, vol. 5, no. 3, pp. 731-738, 2014.

[28] J. P. Dahm, A. Arbor, and K. Fidkowski, "Error estimation and adaptation in hybridized discontinous Galerkin methods," in Proceedings of the 52nd Aerospace Sciences Meeting, The AIAA SciTech, 2014. 


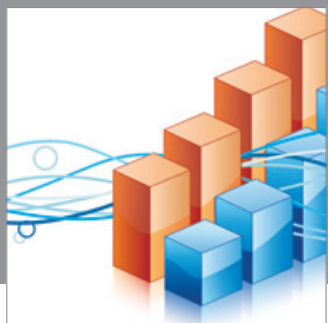

Advances in

Operations Research

mansans

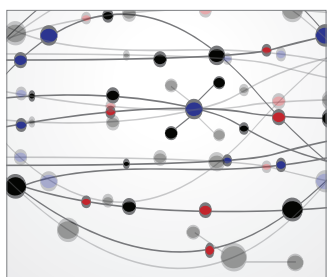

The Scientific World Journal
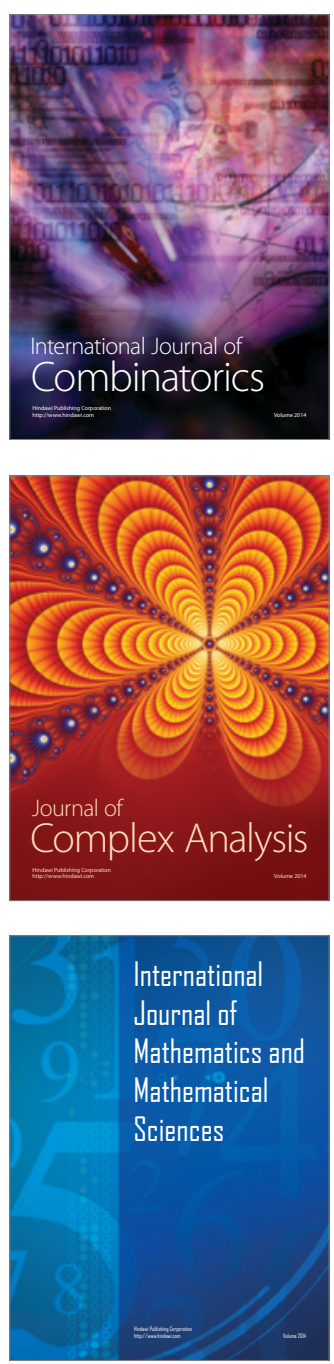
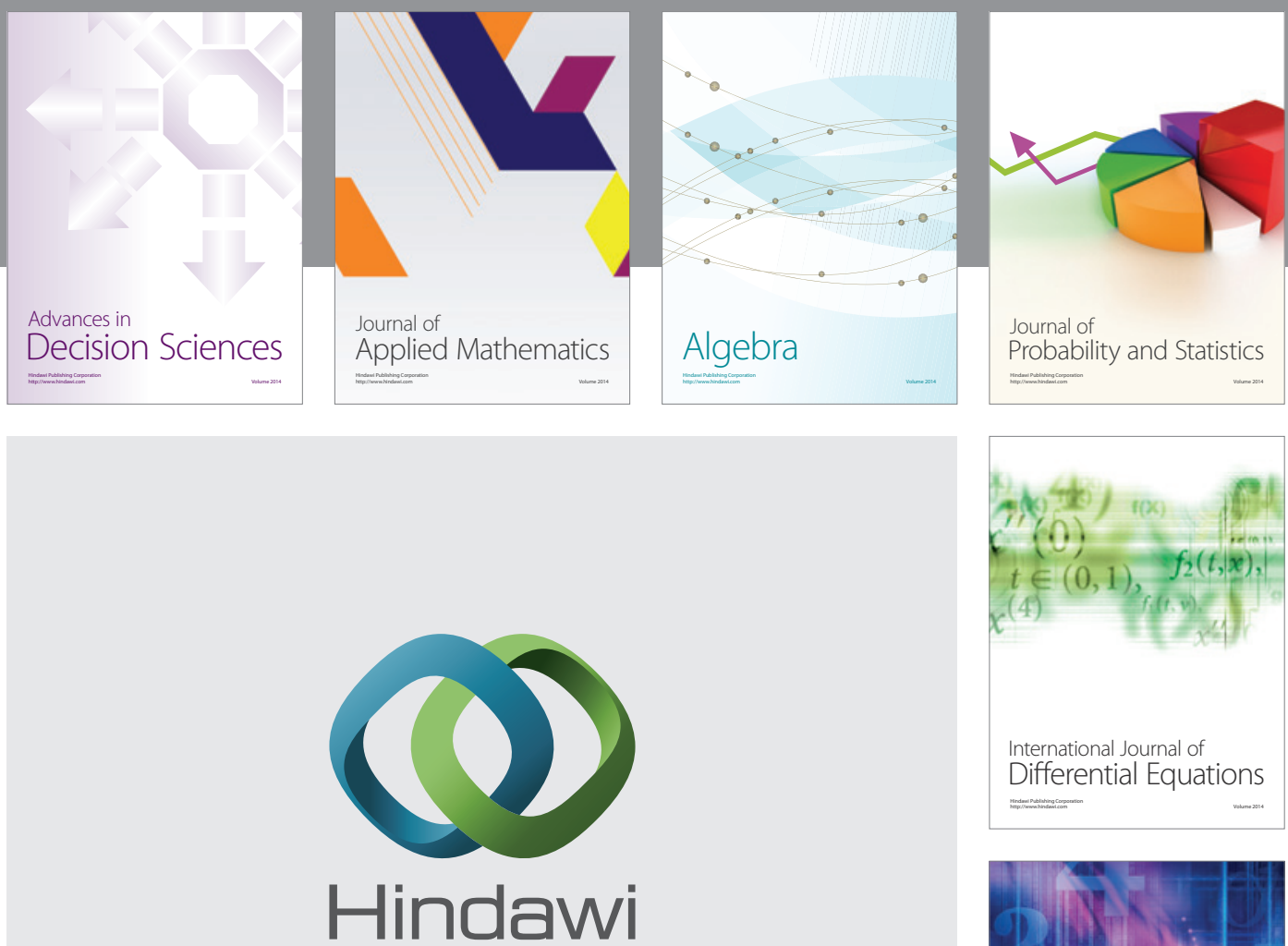

Submit your manuscripts at http://www.hindawi.com
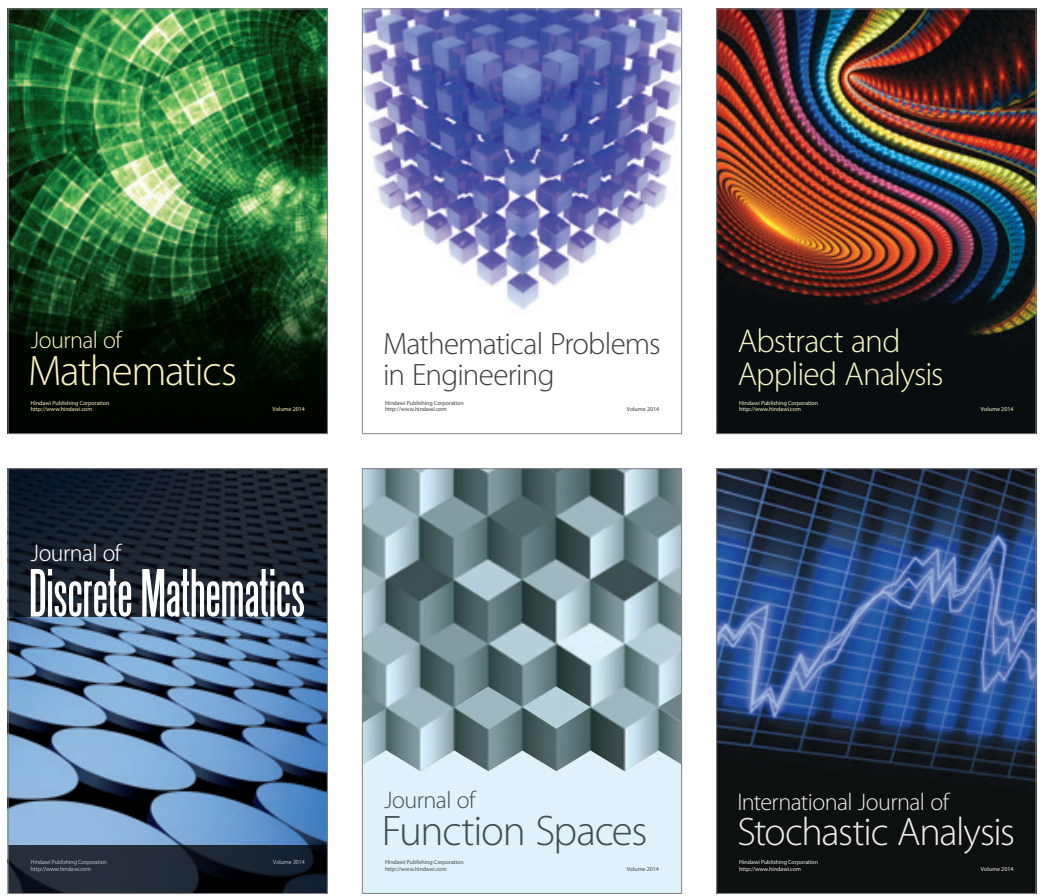

Journal of

Function Spaces

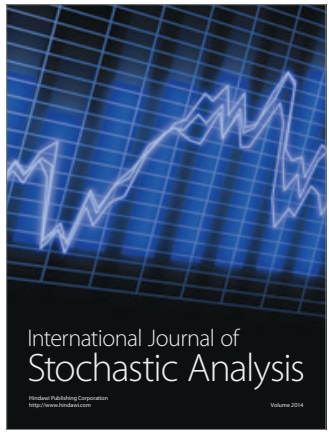

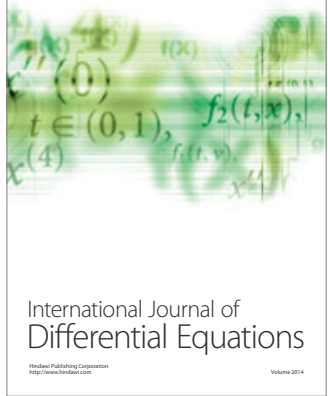
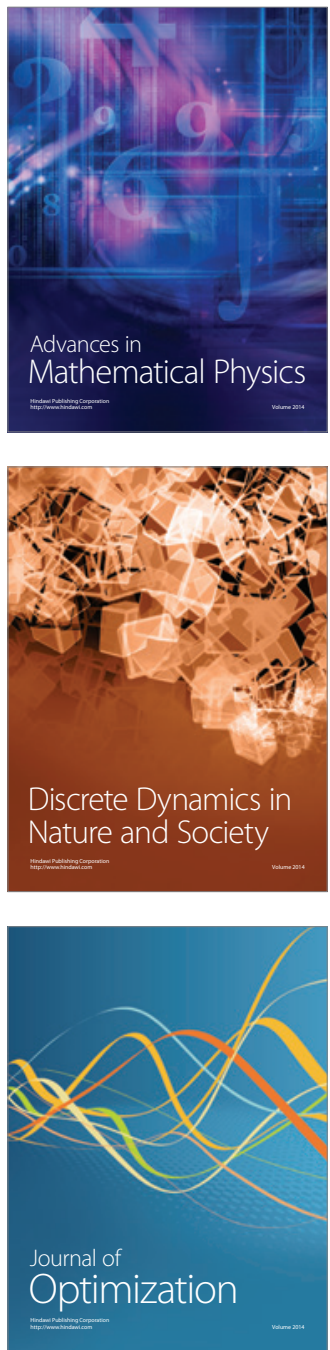